\title{
Complex refractive indices in the TeraHertz domain of samples from atmospheric aerosol sources
}

\author{
J. Bichon ${ }^{1}$, M. Lavancier ${ }^{1}$, D. Petitprez ${ }^{2}$, A. Deguine ${ }^{2}$, D. Hourlier ${ }^{1}$, K. Deboudt ${ }^{3}$, H. Herbin ${ }^{4}$, G. Ducournau ${ }^{1}$, \\ R. Peretti ${ }^{1}$, S. Eliet $^{1}$ \\ ${ }^{1}$ Univ. Lille, CNRS, Centrale Lille, Univ. Polytechnique Hauts-de-France, UMR 8520 IEMN - Institut d'Electronique de \\ Microélectronique et de Nanotechnologie, F-59000 Lille, France \\ ${ }^{2}$ Univ. Lille, CNRS, UMR 8522 - PC2A - Physicochimie des Processus de Combustion et de l'Atmosphère, F-59000 Lille, France \\ ${ }^{3}$ Laboratoire de PhysicoChimie de l'Atmosphère - LPCA, Universite du Littoral Côte d'Opale, Dunkerque, France \\ ${ }^{4}$ Univ. Lille, CNRS, UMR 8518 - LOA - Laboratoire d’Optique Atmosphérique, F-59000 Lille, France
}

\begin{abstract}
We report the TeraHertz (THz) characterization of calcium carbonate $\left(\mathrm{CaCO}_{3}\right)$ particles, one of the major components of desert dust, to determine the real and imaginary parts of their complex refractive indices. The optical characterization is done by TeraHertz Time Domain Spectroscopy (THz-TDS) technique. $\mathrm{CaCO}_{3}$ powders are diluted with High Density PolyEthylene (HDPE) at different concentrations $(0.1 \%$ to $75 \%$ of $\mathrm{CaCO}_{3}$ ) and compressed in pellets. Time-traces are then fitted using the Fit@TDS algorithm developed by Photonic TeraHertz group members, and the complex refractive indices are extracted. We support the optical characterization by a morphological characterization by Scanning Electron Microscopy - Energy Dispersive X-Ray (SEM-EDX) analysis after resuspension of particles and filtration on a polycarbonate $0.2 \mu \mathrm{m}$ pore filter.
\end{abstract}

\section{INTRODUCTION}

$\mathrm{T}$ The Earth is going through a climate change in which variations in the atmospheric environment play a critical role. More precisely, aerosols alter the radiative balance and modify the clouds properties. The quantity of dust particles released per year in the atmosphere is estimated between 1000 and $5000 \mathrm{Tg}$ [1]. These particles in suspension interact with solar and far infrared telluric radiations by absorption and scattering. However, databases have wide gaps regarding refractive indices of materials and aerosols in the $\mathrm{THz}$ range. In a close future, the FORUM (Far-infrared-Outgoing-Radiation Understanding and Monitoring) mission aims to sound the ferinfrared spectral range from space for the first time [2]. It is of decisive importance to know the real and imaginary parts of the complex refractive indices of aerosols in the $\mathrm{THz}$ gap for an accurate environmental evaluation.

THz Time Domain Spectroscopy (THz-TDS) is a valuable technique that measures the time dependent amplitude $\mathrm{E}(\mathrm{t})$ and therefore allows to acquire both the amplitude and phase, simultaneously. One practical advantage of this method is that complex refractive indices of particles are directly calculated without the need of empirical models or Kramers-Kronig analysis [3]. Thereafter, the Fit@TDS software is used on the time-traces to proceed a fitting based on theoretical models and to extract the sample's parameters [4].

The morphological characterization is carried out by Scanning Electron Microscopy - Energy Dispersive X-ray (SEM-EDX) after resuspension of the particles and collection on a $0.2 \mu \mathrm{m}$ pore polycarbonate filter.

\section{RESULtS}

$\mathrm{CaCO}_{3}$ particles (Riedel-deHaën 31208 - calcite) were resuspended and filtered on nuclepore polycarbonate filter membranes with uniform hole sizes of $0.2 \mu \mathrm{m}$ for subsequent SEM-EDX examination. Collected particles were explored through the back-scattered electron and EDX detectors at the LPCA and through the secondary electron detector at the IEMN. The potential chemical contamination of samples from the resuspension process was discarded by EDX. For a good data representativeness, the pictures of several hundreds of particles were automatically acquired with the Esprit 2.0 software for morphological analysis.

The obtained secondary electron detector micrographs of typical particles are shown in Figure 1. Our results demonstrated that the equivalent diameter of resuspended particles was between $0.30 \mu \mathrm{m}$ and $5.43 \mu \mathrm{m}$. The particles were mostly aggregated and non-rounded.
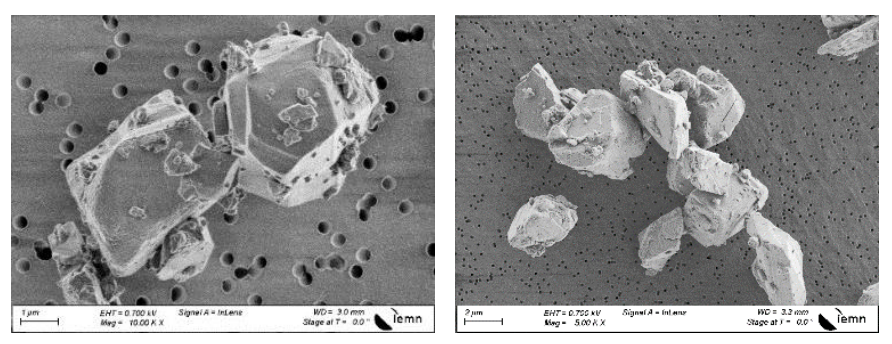

Fig. 1. Secondary electron detector micrographs of $\mathrm{CaCO}_{3}$ particles for a time of resuspension and collection on filter of 10 minutes. The $0.2 \mu \mathrm{m}$ pores of the filter are visible on the background.

Before studying the primary sources of aerosols in the form of particles suspended in the air via a dedicated spectrometer, a first step is the study in the form of pelletized material. $\mathrm{CaCO}_{3}$ powder were diluted with polyethylene (HDPE - Aldrich Chemistry 43472-100G -Lot MKCG2724 ; 40-48 $\mu \mathrm{m}$ particle size) and compressed in pellets with a diameter of $13 \mathrm{~mm}$ and a thickness of approximately $500 \mu \mathrm{m}$. Mass percentages of $\mathrm{CaCO}_{3}$ were ranging from $0.1 \%$ to $75 \%$. The hydration rate was verified to $0 \%$ and the purity to $99.32 \%$ by thermogravimetric analysis coupled with mass spectrometry [5]. The THz timetraces were recorded with a commercial Menlo systems THzTDS and analyzed with the Fit@TDS algorithm.

Results were consistent with previous research [6] and confirmed that calcite powder had a wide absorption band around $3 \mathrm{THz}$. The refractive index was almost entirely imaginary at the resonant frequency. In addition, we showed that $\mathrm{PE}$ contribution was very low in the $\mathrm{THz}$ region as both the real and imaginary parts were flat.

Fig. 2 and Fig. 3 show the imaginary and the real refractive index results from an initial data processing of pure $\mathrm{PE}$ and $10 \%$ $\mathrm{CaCO}_{3}$ pellets time-traces with the Boltzmann model. 

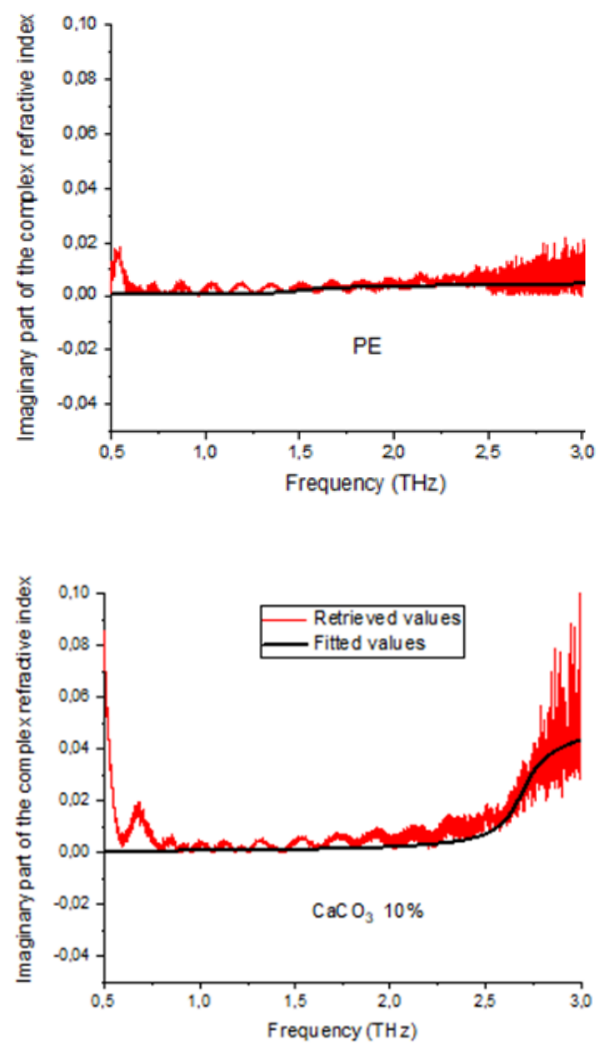

Fig. 2. Imaginary parts of $10 \% \mathrm{CaCO}_{3}$ diluted with $\mathrm{PE}$ and pure PE pellets. The retrieved values are in red. The solid black lines represent the fits to the data by the Boltzmann model.
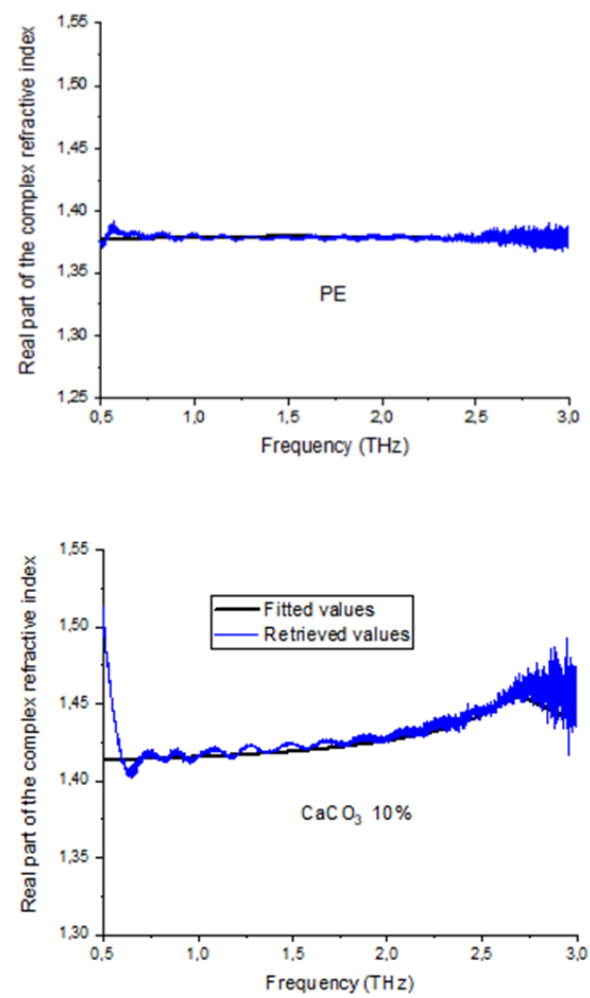

Fig. 3. Real parts of $10 \% \mathrm{CaCO}_{3}$ diluted with $\mathrm{PE}$ and pure PE pellets. The retrieved values are in blue. The solid black lines represent the fits to the data by the Boltzmann model.

\section{SUMMARY}

In this study, we aimed to determine, for the first time and simultaneously in a single experiment, the real and imaginary parts of the complex refractive indices in the $\mathrm{THz}$ domain of a typical material source of primary atmospheric aerosols. The optical properties were supported with a morphological overview using SEM-EDX technique. Further research is needed to separate $\mathrm{CaCO}_{3}$ response from PE response by implementing the Bruggeman model [7] that allows the study of material mixtures in the pellets by ensuring that the permittivity is equal to that of the effective mixture. However, these results should be replicated in a study where the particles would be resuspended. In addition, a transportable bench dedicated to the study of resuspended particles in the $\mathrm{THz}$ domain is currently being developed. In the future, the $\mathrm{THz}$ bench will be coupled to Infrared, Visible and Ultraviolet spectrometers [8] to achieve an on-line and simultaneous measurement on resuspended particles. Finally, desert dust and volcanic ash particles will be considered for further investigation.

\section{ACKNOWLEDGMENTS}

We sincerely thank our colleagues Valentine Bizet from the PC2A for the resuspension of particles and Christophe Boyaval from the IEMN for the SEM analysis. Funding for the present work was provided by the French National Research Agency (ANR-20-CE42-0002 STEPSON). The authors very much appreciated the support by the CaPPA project (Chemical and Physical Properties of the Atmosphere) funded by the French National Research Agency (ANR) through the PIA (Programme d'Investissement d'Avenir) under contract « ANR-11-LABX-0005-01 ». The authors would also like to thank the "Hauts-de-France" Region and the Ministry of Higher Education and Research (CPER IRenE), as well as the European Fund for Regional Economic Development, for their financial support.

\section{REFERENCES}

[1]. A. Petzold, B. Kärcher, U. Schumann (ed), "Aerosols in the atmosphere", Atmospheric Physics, p38 \& p48, 2012.

[2]. FORUM (Far-Infrared-Outgoing-Radiation Understanding and Monitoring) mission website: https://www.forum-ee9.eu/

[3]. H. Herbin et al, "New approach for the determination of aerosol refractive indices - Part I: Theoretical bases and numerical methodology", Journal of Quantitative Spectroscopy and Radiative Transfer 200, March 2017.

[4]. R. Peretti, et al, "THz-TDS time-trace analysis for the extraction of material and metamaterial parameters", IEEE transactions on Terahertz Science and Technology, 9, 136, 2019. Software available on: https://github.com/THzbiophotonics/Fit-TDS.

[5]. D. Hourlier, "Thermal decomposition of calcium oxalate: beyond appearances", Journal of Thermal Analysis and Calorimetry, 136:2221-2229, 2019.

[6]. M. Mizuno et al, "Analysis of calcium carbonate for differentiating between pigments using TeraHertz spectroscopy", Journal of the European Optical Society-Rapid Publications, vol 4, 2009.

[7]. M. H. Boyle, 'The electrical properties of heterogeneous mixtures containing an oriented spheroidal dispersed phase', Colloid Polym. Sci., vol. 263, no. 1, pp. 51-57, Jan. 1985

[8]. A. Deguine, et al, "Complex refractive index of volcanic ash aerosol in the infrared, visible, and ultraviolet", Applied Optics, Vol. 59, No. 4, 1 February 2020. 